International Journal of Pure and Applied Mathematics

Volume 87 No. 2 2013, 247-260

ISSN: 1311-8080 (printed version); ISSN: 1314-3395 (on-line version)

url: http://www.ijpam.eu

doi: http://dx.doi.org/10.12732/ijpam.v87i2.5

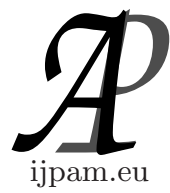

\title{
ON THE THERMAL VOLTAGE SIGNAL IN A VIRTUAL NANOCONDUCTOR
}

\author{
Eugen Grycko ${ }^{1}$, Werner Kirsch ${ }^{2}$, Tobias Mühlenbruch ${ }^{3}$ \\ ${ }^{1,2,3}$ Department of Mathematics and Computer Science \\ University of Hagen \\ Universitätsstrasse 1, D-58084 Hagen, GERMANY
}

\begin{abstract}
We consider the electronic gas in a virtual conductor which is described in terms of a modified Drude model. The attractiveness of this model has been rediscovered in the computer era when it has been recognized that a dynamics of the electrons can be efficiently implemented enabling us to carry out simulation experiments.

Nonparametric statistical tools for the evaluation of the experiments are introduced and motivated by consistency results.

It turns out that the thermal voltage in a virtual nanoconductor can be surprisingly high. Based on the statistical evaluation we propose the stationary Ornstein-Uhlenbeck process for the description of the thermal voltage as function of time; estimates of the parameters of the process are reported and discussed.
\end{abstract}

AMS Subject Classification: 60G10, 60G15, 60G99, 62G07, 62G20

Key Words: $\alpha$-mixing, estimator, system identification

\section{Introduction}

Recently we have perceived some interest in thermal noise of electric charge

Received: February 15, 2013

(C) 2013 Academic Publications, Ltd.

$\S$ Correspondence author url: www.acadpubl.eu 
carriers cf. [3], [4] and [5]. Statistical Thermodynamics offers a natural access to this kind of phenomena. In particular, the Drude model (cf. [13]) is an attractive possibility for computer based exploration of electrodynamic properties that result from thermal movement of charge carriers. We exemplify this idea for a virtual nanoconductor which can be analyzed under a reasonable computational effort.

We assume that the thermal voltage signal is a trajectory of a stationary and $\alpha$-mixing process; these assumptions specify a nonparametric statistical model in which the autoregression function and the marginal distribution are to be estimated. The estimation of the marginal density of a stationary process has been extensively studied during the last decades (cf. [8], [9], [10] and literature cited therein). To give the reader an impression of methodic justification of the kernel density estimator, we formulate and prove a weak consistency theorem which is based on mild conditions on the stochastic process; the interested reader is referred to [10] where a stronger result requiring a more sophisticated proof can be found.

The paper is organized as follows. In Section 2 we introduce and comment " $\alpha$-mixing" and "stationarity" as properties of stochastic processes that are reasonable for describing certain physical phenomena and imply consistency of a natural estimator for the autocovariance. In Section 3 the kernel density estimator is motivated and proposed for the estimation of the marginal distribution in an appropriate (nonparametric) class of stationary processes. In Section 4 we remark that an equidistant discrete sampling from a stationary Ornstein-Uhlenbeck process leads to a discrete time autoregressive process. In Section 5 the modified Drude model describing the electronic gas in a virtual conductor is presented together with a possibility of simulating its dynamics; inspired by standard facts from Electrodynamics it is pointed out how to extract the virtual thermal voltage values during the computational process. In Section 6 the computer experiment is specified; we also report and comment its outcome applying the statistical estimators motivated in Section 2 and 3.

\section{Stationarity and Mixing in Discrete Time}

Let $(\Omega, \mathcal{A}, P)$ be a probability space and $\left(X_{n}\right)_{n=1}^{\infty}$ a stochastic process with discrete time; this means that $\left(X_{n}\right)_{n=1}^{\infty}$ is a sequence of real random variables $X_{n}: \Omega \rightarrow \mathbb{R}$. We call $\left(X_{n}\right)$ stationary if the distribution of the random vectors $\left(X_{n}, \ldots, X_{n+j}\right)$ is independent of $n$ for $j=1,2, \ldots$ 
Remark 2.1. The assumption of stationarity plays a role for the description of physical systems whose microscopic state fluctuates and whose macroscopic state does not change in time.

Let $\left(\alpha_{n}\right)$ be a real sequence such that

$$
|P(A \cap B)-P(A) \cdot P(B)| \leq \alpha_{n}
$$

for all $A \in \sigma\left(X_{1}, \ldots, X_{k}\right)$, for all $B \in \sigma\left(X_{k+n}, X_{k+n+1}, \ldots\right)$ and for $n, k=1,2, \ldots$ where $\sigma\left(X_{1}, \ldots, X_{k}\right)$ denotes the $\sigma$-algebra generated by the random vector $\left(X_{1}, \ldots, X_{k}\right)$. The stochastic process is called $\alpha$-mixing if $(2.1)$ and

$$
\lim _{n \rightarrow \infty} \alpha_{n}=0
$$

hold for a sequence $\left(\alpha_{n}\right)$.

Remark 2.2. $\alpha$-mixing implies that the microstates of the system described by the stochastic process are nearly stochastically independent if they are observed at distant time points.

The numbers $\alpha_{n}$ satisfying (2.1) can be used for establishing bounds on the covariance of appropriate random variables.

For a real random variable $Y: \Omega \rightarrow \mathbb{R}$ define

$$
\|Y\|_{\infty}:=\sup _{\omega \in \Omega}|Y(\omega)|
$$

Lemma 2.3. Let the process $\left(X_{n}\right)$ be $\alpha$-mixing and a sequence $\left(\alpha_{n}\right)$ may satisfy (2.1) and (2.2). Let $Y, Z: \Omega \rightarrow \mathbb{R}$ be two random variables where $Y$ is $\sigma\left(X_{1} \ldots, X_{k}\right)$ - and $Z$ is $\sigma\left(X_{k+n}, X_{k+n+1}, \ldots\right)$-measurable for some $k$ and some $n$.

(1) An upper bound for the covariance $\operatorname{cov}(Y, Z)$ is given by

$$
|\operatorname{cov}(Y, Z)| \leq 4 \cdot\|Y\|_{\infty} \cdot\|Z\|_{\infty} \cdot \alpha_{n}
$$

(2) An upper bound for the covariance is given by

$$
|\operatorname{cov}(Y, Z)| \leq 8 \cdot\left(1+\mathbb{E}\left(|Y|^{4}\right)+\mathbb{E}\left(|Z|^{4}\right)\right) \cdot \alpha_{n}^{1 / 2}
$$

where $\mathbb{E}$ denotes expectation.

Proof. Lemma 2.3 is a reformulation of Lemma 2 and Lemma 3 in [2], p. 365 .

Let us now suppose that $\left(X_{n}\right)$ is a stationary and $\alpha$-mixing process and that 
$X_{1}$ is centered $\left(\mathbb{E}\left(X_{1}\right)=0\right)$ and square-integrable; under these assumptions the expression $\operatorname{cov}\left(X_{n}, X_{n+k}\right)$ is finite and independent of $n$ for $k=0,1, \ldots$

Put

$$
\gamma(k):=\operatorname{cov}\left(X_{1}, X_{1+k}\right) \quad(k=0,1, \ldots) .
$$

The sequence $(\gamma(k))_{k=0}^{\infty}$ is called the autocovariance function of the process $\left(X_{n}\right)$.

Suppose that we would like to estimate $\gamma(k)$ from the observations $X_{1}, X_{2}, \ldots$ Since $\mathbb{E}\left(X_{n}\right)=0$ for $n=1,2, \ldots$, a natural estimator is given by:

$$
\widehat{\gamma}_{n}(k):=\frac{1}{n-k} \cdot \sum_{j=1}^{n-k} X_{j} \cdot X_{j+k}
$$

for $n=1,2, \ldots$.

Note that estimator $\widehat{\gamma}_{n}(k)$ is unbiased for $n=1,2, \ldots$. Now we formulate general (nonparametric) conditions that are sufficient for weak consistency of $\left(\widehat{\gamma}_{n}(k)\right)_{n=1}^{\infty}$.

Lemma 2.4. Suppose that the process $\left(X_{n}\right)$ is stationary and $\alpha$-mixing where $\mathbb{E}\left(X_{1}\right)=0$. Fix a nonnegative integer $k$ and let us assume that

$$
C:=\sup _{l=0}^{\infty} \mathbb{E}\left(\left|X_{1+l} X_{1+k+l}\right|^{4}\right)<\infty .
$$

holds. Then

$$
\widehat{\gamma}_{n}(k) \rightarrow \gamma(k) \quad \text { in probability for } n \rightarrow \infty \text {. }
$$

Proof. Due to Chebyshev inequality it suffices to prove that

$$
\lim _{n \rightarrow \infty} \operatorname{Var}\left(\widehat{\gamma}_{n}(k)\right)=0
$$

holds for the sequence of variances.

Let $\left(\alpha_{n}\right)$ be a sequence satisfying (2.1) and (2.2). A standard reasoning using the stationarity of $\left(X_{n}\right)$ implies that

$$
\begin{aligned}
\operatorname{Var}\left(\widehat{\gamma}_{n}(k)\right)= & \frac{1}{(n-k)^{2}} \cdot \sum_{j=1}^{n-k} \operatorname{Var}\left(X_{j} X_{j+k}\right) \\
& +\frac{2}{(n-k)^{2}} \cdot \sum_{1 \leq i<j \leq n-k} \operatorname{cov}\left(X_{i} X_{i+k}, X_{j} X_{j+k}\right) \\
= & \frac{1}{n-k} \operatorname{Var}\left(X_{1} X_{1+k}\right)
\end{aligned}
$$




$$
\begin{aligned}
& +\frac{2}{(n-k)^{2}} \cdot \sum_{l=1}^{n-k-1}(n-k-l) \cdot \operatorname{cov}\left(X_{1} X_{k}, X_{1+l} X_{1+k+l}\right) \\
\leq & \frac{1}{n-k} \operatorname{Var}\left(X_{1} X_{1+k}\right) \\
& +\frac{2}{n-k} \sum_{l=1}^{n-k-1}\left|\operatorname{cov}\left(X_{1} X_{1+k}, X_{1+l} X_{1+k+l}\right)\right| .
\end{aligned}
$$

According to Lemma 2.3(2) we have

$$
\left|\operatorname{cov}\left(X_{1} X_{1+k}, X_{1+l} X_{1+k+l}\right)\right| \leq 8 \cdot(1+2 C) \cdot \alpha_{l-k}^{1 / 2}
$$

for $l>k$. This completes the proof in view of (2.2).

Remark 2.5. The upper bounds for the variance of estimator $\widehat{\gamma}_{n}(k)$ presented in the proof of Lemma 2.4 depend on lag $k$; the proof suggests that sample size $n$ required for estimating covariance $\gamma(k)$ should be much higher than lag $k$ :

$$
n>>k \text {. }
$$

Figure 2.1 in [6] illustrates the consequences of violation of (2.4) in a more general context.

\section{An Estimator for the Marginal Density}

Stationarity and $\alpha$-mixing are mild conditions imposed on a stochastic process $\left(X_{n}\right)$; they are also physically plausible in the context of modeling numerous phenomena (cf. Remarks 2.1 and 2.2). In the present section we propose the kernel density estimator for a statistical exploration of the marginal distribution of a stationary and $\alpha$-mixing process. The kernel density estimator is an approved nonparametric procedure for estimating the Lebesgue density of the distribution of observables; in our context it can be defined by

$$
\widehat{f}_{n}(x):=\frac{1}{n h(n)} \cdot \sum_{j=1}^{n} K\left(\frac{x-X_{n}}{h(n)}\right) \quad(x \in \mathbb{R})
$$

for $n=1,2, \ldots$ where $K: \mathbb{R} \rightarrow \mathbb{R}_{+}$is a Lebesque density of a probability distribution on the real line (kernel) and $h(n)$ is a sequence of bandwidths. To motivate the application of $\widehat{f}_{n}$ for the statistical access to the thermal voltage signal we formulate and prove a weak consistency result for the kernel density estimator. 
Theorem 3.1. Let $\left(X_{n}\right)$ be a stationary and $\alpha$-mixing process where a sequence $\left(\alpha_{n}\right)$ satisfies (2.1) and

$$
\sum_{n=1}^{\infty} \alpha_{n}<\infty
$$

The distribution of $X_{1}$ may have a continuous and bounded Lebesgue density $f: \mathbb{R} \rightarrow \mathbb{R}_{+}$. Let the kernel $K$ be a bounded density of a probability measure. Put

$$
h(n):=\frac{c}{n^{\beta}} \quad \text { for } n=1,2, \ldots
$$

where $c>0$ is a constant and $0<\beta<1 / 2$ an exponent. Then

$$
\widehat{f}_{n}(x) \rightarrow f(x) \quad \text { in probability for } n \rightarrow \infty
$$

for all $x \in \mathbb{R}$.

Proof. Fix an arbitrary $x \in \mathbb{R}$. Obviously

$$
\mathbb{E}\left(\widehat{f}_{n}(x)\right)=\frac{1}{h(n)} \int_{\mathbb{R}} K\left(\frac{x-\xi}{h(n)}\right) f(\xi) d \xi
$$

it follows that $\left(\widehat{f}_{n}(x)\right)$ is asymptotically unbiased:

$$
\lim _{n \rightarrow \infty} \mathbb{E}\left(\widehat{f}_{n}(x)\right)=f(x)
$$

(cf. Theorem 9.8 in [16]). Therefore it remains to show that

$$
\lim _{n \rightarrow \infty} \operatorname{Var}\left(\widehat{f}_{n}(x)\right)=0
$$

holds.

Now, standard computation applying the stationarity of $\left(X_{n}\right)$ yields

$$
\begin{aligned}
\operatorname{Var}\left(\widehat{f}_{n}(x)\right) \\
=\frac{1}{(n h(n))^{2}} \cdot \sum_{j=1}^{n} \operatorname{Var}\left(K\left(\frac{x-X_{j}}{h(n)}\right)\right) \\
\quad+\frac{2}{(n h(n))^{2}} \cdot \sum_{1 \leq i<j \leq n} \operatorname{cov}\left(K\left(\frac{x-X_{i}}{h(n)}\right), K\left(\frac{x-X_{j}}{h(n)}\right)\right) \\
=\frac{1}{n h(n)^{2}} \operatorname{Var}\left(K\left(\frac{x-X_{1}}{h(n)}\right)\right)
\end{aligned}
$$




$$
\begin{aligned}
& +\frac{2}{(n h(n))^{2}} \cdot \sum_{l=1}^{n-1}(n-l) \cdot \operatorname{cov}\left(K\left(\frac{x-X_{1}}{h(n)}\right), K\left(\frac{x-X_{1+l}}{h(n)}\right)\right) \\
\leq & \frac{\|K\|_{\infty}^{2}}{n \cdot h(n)^{2}}+\frac{2}{n h(n)^{2}} \sum_{l=1}^{n-1}\left|\operatorname{cov}\left(K\left(\frac{x-X_{1}}{h(n)}\right), K\left(\frac{x-X_{1+l}}{h(n)}\right)\right)\right| .
\end{aligned}
$$

Application of Lemma 2.3(1) entails the inequality

$$
\operatorname{Var}\left(\widehat{f}_{n}(x)\right) \leq \frac{\|K\|_{\infty}^{2}}{n h(n)^{2}}+\frac{8 \cdot\|K\|_{\infty}^{2}}{n h(n)^{2}} \cdot \sum_{l=1}^{\infty} \alpha_{l}
$$

which in view of (3.1) proves (3.2).

Example 3.2. (Autoregressive Process) Let $\left(\varepsilon_{j}\right)_{j=-\infty}^{\infty}$ be an i.i.d. sequence of random variables distributed according to the normal distribution with mean 0 and variance $\sigma^{2}>0$. Let $0<a<1$ be a number. Put

$$
X_{n}:=\sum_{j=0}^{\infty} a^{j} \cdot \varepsilon_{n-j}
$$

for $n \in \mathbb{Z}$ where $\mathbb{Z}$ denotes the set of integers. Obviously, $\left(X_{n}\right)_{n=-\infty}^{\infty}$ is a stationary Gaussian process whose autocovariance function can be computed according to:

$$
\begin{aligned}
\gamma(l) & =\operatorname{cov}\left(X_{n}, X_{n+l}\right) \\
& =\sum_{i=0}^{\infty} \sum_{j=0}^{\infty} a^{i} a^{j} \cdot \mathbb{E}\left(\varepsilon_{n-i} \cdot \varepsilon_{n+l-j}\right) \\
& =\sum_{i=0}^{\infty} a^{i} \cdot a^{l+i} \cdot \sigma^{2}=\frac{\sigma^{2} \cdot a^{l}}{1-a^{2}}
\end{aligned}
$$

for $n \in \mathbb{Z}$ and $l=0,1, \ldots$ It follows that

$$
\gamma(l)=\frac{\sigma^{2} \cdot a^{|l|}}{1-a^{2}}
$$

holds for $l \in \mathbb{Z}$. The sumability of $(\gamma(l))_{l=-\infty}^{\infty}$ implies that the spectral density $f$ of $\left(X_{n}\right)$ is given by

$$
f(\lambda):=\frac{1}{2 \pi} \sum_{k=-\infty}^{\infty} \gamma(k) \cdot \exp (-i k \lambda) \quad(\lambda \in[-\pi, \pi])
$$


standard calculation using (3.3) entails that

$$
f(\lambda)=\frac{\sigma^{2}}{2 \pi\left(1-2 a \cos \lambda+a^{2}\right)} \quad(\lambda \in[-\pi, \pi])
$$

holds. Theorem 5 on p. 67 in [15] implies now that the autoregressive process $\left(X_{n}\right)$ satisfies the assumptions of Lemma 2.4 and of Theorem 3.1. Therefore estimator $\left(\widehat{\gamma}_{n}\right)$ defined in Section 2 admits consistent estimation of the autocovariance function $\gamma$ and kernel estimator $\left(\widehat{f}_{n}\right)$ is weakly consistent for the marginal density of $\left(X_{n}\right)$.

\section{Equidistant Sampling from the Ornstein-Uhlenbeck Process}

Let $\left(Y_{t}\right)_{t \in \mathbb{R}}$ be a centered and stationary Ornstein-Uhlenbeck process on a probability space $(\Omega, \mathcal{A}, P)$. This means that

$$
\mathbb{E}\left(Y_{t}\right)=0 \quad(t \in \mathbb{R})
$$

holds and that

$$
\Gamma(s):=\operatorname{cov}\left(Y_{t}, Y_{t+s}\right)=b \cdot \exp (-c \cdot|s|)
$$

is independent of $t$ for all $s \in \mathbb{R}$ where $b$ and $c$ are positive constants (parameters of the process); process $\left(Y_{t}\right)_{t \in \mathbb{R}}$ is Gaussian which implies that it is well defined by the covariance structure (4.2) and by the condition of continuity of paths, cf. [1].

Let us now interpret $\left(Y_{t}\right)_{t \in \mathbb{R}}$ as a model for a continuous time signal which is sampled at equidistant discrete time points $k \cdot \Delta t, k \in \mathbb{Z}$ by a digital device. Put

$$
X_{n}:=Y_{n \cdot \Delta t} \quad(n \in \mathbb{Z}) .
$$

Obviously, $\left(X_{n}\right)_{n \in \mathbb{Z}}$ is a stationary and centered Gaussian process in discrete time; its autocovariance function is given by:

$$
\gamma(l)=\operatorname{cov}\left(X_{0}, X_{l}\right)=b \cdot \exp (-c \cdot \Delta t \cdot|l|) \quad(l \in \mathbb{Z})
$$

which corresponds to the autocovariance function (3.3) in Example 3.2. We conclude by Consistency Theorem of Daniell and Kolmogorov (cf. [1], Theorem 35.3) that the discrete extract $\left(X_{n}\right)$ from $\left(Y_{t}\right)$ is the autoregressive process presented in Section 3 where parameters $a$ and $\sigma$ in (3.3) can be adjusted to the parameters $b$ and $c$ of the Ornstein-Uhlenbeck process $\left(Y_{t}\right)_{t \in \mathbb{R}}$, cf. (4.3). 


\section{The Modified Drude Model for the Electronic Gas}

Let us consider a 3-dimensional container $C$ which is modeled by

$$
C:=[0, L] \times[-w / 2,+w / 2]^{2} \subset \mathbb{R}^{3}
$$

where $L, w>0$ denote the edge lengths. We inject $N$ mass points of mass $m>0$ into $C$ according to the uniform distribution. The initial velocities $v^{(1)}(0), \ldots, v^{(N)}(0) \in \mathbb{R}^{3}$ of the points are generated according to the centered Gaussian distribution $N\left(0, \sigma^{2} \cdot I_{3}\right)$ with mean $0 \in \mathbb{R}^{3}$ and covariance matrix $\sigma^{2} \cdot I_{3}$ where $I_{3}$ denotes the $3 \times 3$-identity matrix; parameter $\sigma$ can be interpreted thermally by

$$
\sigma=\left(\frac{k_{B} \cdot T}{m}\right)^{1 / 2}
$$

where $k_{B}=1.380662 \cdot 10^{-23} \mathrm{~J} / \mathrm{K}$ and $T>0$ denote Boltzmann constant and temperature of the system, respectively, cf. [12].

Let the system of mass points (gas) evolve according to the Newtonian dynamics entailing that the micro-constituents do not mutually interact and are reflected at the walls of container $C$ at appropriate time points. The system can be interpreted as a kinetic model of the ideal gas (cf. [11], Section 2.3). This model can be implemented in a computer program enabling us to compute a trajectory

$$
\left(x^{(1)}(t), \ldots, x^{(N)}(t) ; v^{(1)}(t), \ldots, v^{(N)}(t)\right) \quad\left(t \in \mathbb{R}_{+}\right)
$$

where $x^{(j)}(t)$ and $v^{(j)}(t)$ denotes the position and velocity vector of $j^{\text {th }}$ mass point at time $t$, respectively, $j=1, \ldots, N$.

Fixing mass $m=9.109534 \cdot 10^{-31} \mathrm{~kg}$ of the micro-constituents implies that the system can be viewed as a gas of electrons of charge $e=-1.60219 \cdot 10^{-19} \mathrm{C}$ confined to a virtual conductor in the sense of a modified Drude model (cf. [13] and [3]) in which the Coulomb repulsion between the electrons is neglected.

The electric potential $V$ of the electronic gas as function of position vector $y$ and time $t$ is given by

$$
V(y, t):=\frac{e}{4 \pi \varepsilon_{0}} \cdot \sum_{j=1}^{N} \frac{1}{\left|y-x^{(j)}(t)\right|}
$$

where $\varepsilon_{0}=8.8543 \cdot 10^{-12} \mathrm{As} / \mathrm{Vm}$ and $|$.$| denotes the permittivity of vacuum$ and the Euclidean norm, respectively; cf. [7] and [14]. Accordingly, the thermal voltage signal $U$ between the ends of the conductor is given by

$$
U(t):=V((L, 0,0), t)-V((0,0,0), t) \quad\left(t \in \mathbb{R}_{+}\right) .
$$


Example 5.1. A typical size of the electronic gas which can be reasonably processed on a contemporary computer, is $N=10^{4}$. Density $\varrho$ of the electronic gas in copper is given by

$$
\varrho=8.4723 \cdot 10^{28} \mathrm{~m}^{-3}
$$

cf. [4]. This means that under the assumption that the virtual copper probe is a cube $(L=w)$, the typical edge length is $L=4.90531 \cdot 10^{-9} \mathrm{~m}$ which in our context justifies the term "nanoconductor".

\section{The Computer Experiment and its Outcome}

Our statistical access to voltage signal $U$ in a virtual nanoconductor is based on the generation of a trajectory (5.2) in the phase space during a simulation experiment; in the course of the computational process the values $U(\Delta t \cdot n)$, $n=0,1, \ldots$ are sampled where $\Delta t=10^{-16} \mathrm{~s}$ is fixed. The nonparametric statistical procedures introduced in Sections 2 and 3 are applied to the sample.

Let us specify the input data for the computer experiment. We fix the number $N=10^{4}$ of electrons to be considered. Put $L=w=4.90531 \cdot 10^{-9} \mathrm{~m}$ for the length and width of nanoconductor $C$ and $T=300 \mathrm{~K}$ for the temperature. The initial positions of the electrons are generated according to the uniform distribution over $C$ and the initial velocity vectors according to Gaussian distribution $N\left(0, \sigma^{2} \cdot I_{3}\right)$ with parameter $\sigma$ defined by (5.1). The Newtonian dynamics is imposed on the electronic gas whose microstate (5.2) evolves; during the experiment the thermal voltage values $U(\Delta t \cdot n), n=0,1, \ldots, M=10^{6}$ are stored. For the statistical analysis of the stored data we assume the nonparametric statistical model as specified in Sections 2 and 3.

Figure 1 shows a typical discrete sample of the voltage signal and confirms the appropriateness of our choice of $\Delta t$.

The noisy curve in Figure 2 corresponds to the kernel density estimate of the marginal distribution of the thermal voltage introduced in Section 3 while the smooth curve represents the centered Gaussian distribution whose variance has been estimated by $\widehat{\gamma}_{M}(0)$, cf. Section 2. Figure 2 confirms the Gaussianity of the stochastic process modeling the voltage signal.

The noisy curve in Figure 3 is the nonparametric estimate of the autocovariance function of the thermal voltage observed in the course of the experiment. The smooth curve is the parametric fit (cf. (4.3)) to the nonparametric estimate. The diagram suggests that the parametric model approximates the 


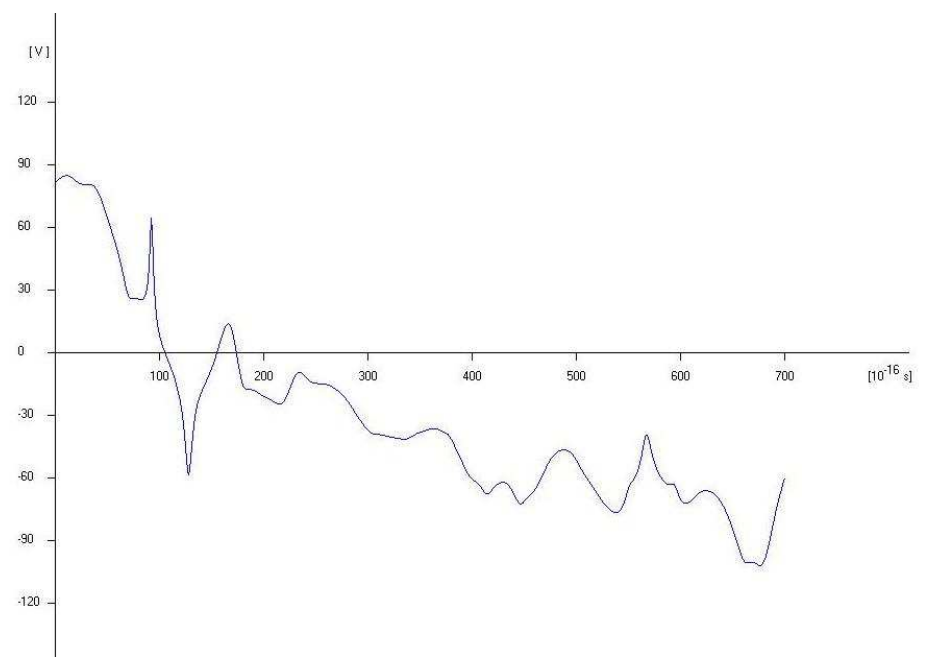

Figure 1: Observed voltage signal

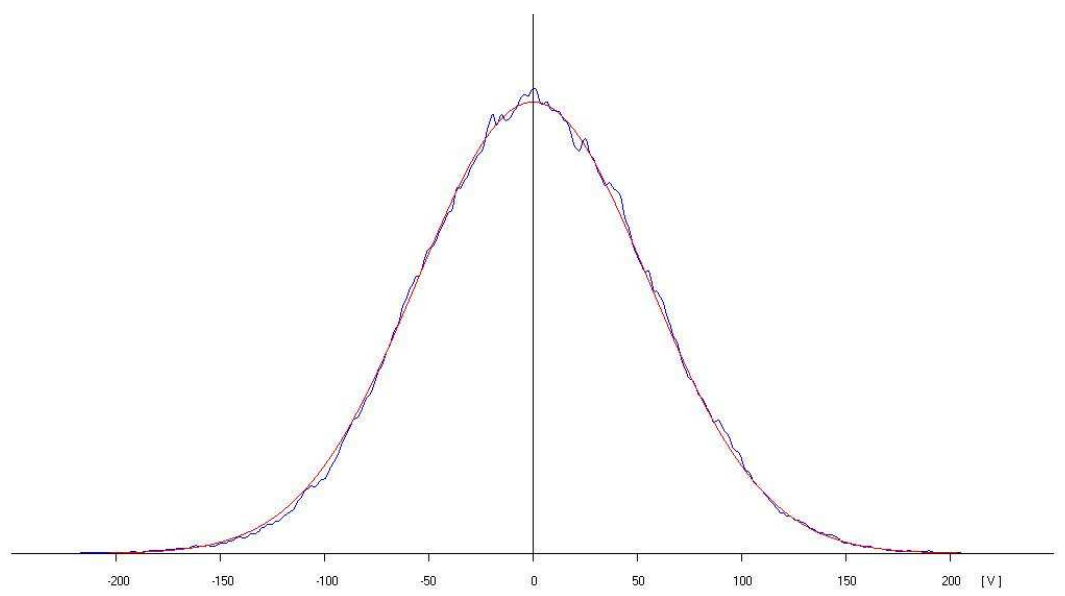

Figure 2: Parametric and nonparametric density estimates

nonparametric estimate by $\widehat{\gamma}_{M}$; the slight discrepancy between the curves can be interpreted as a consequence of statistical risk inherent in estimator $\widehat{\gamma}_{M}$.

The estimates of parameters $b$ and $c$ are

$$
\widehat{b}=3.0544 \cdot 10^{3} \mathrm{~V}^{2} \quad \text { and } \quad \widehat{c}=5.4375 \cdot 10^{13} \mathrm{~s}^{-1} .
$$

They indicate a surprisingly high average thermal voltage in our virtual nanoconductor which can be approximated by the square root of $\widehat{b}$. Estimate $\widehat{c}$ can be 


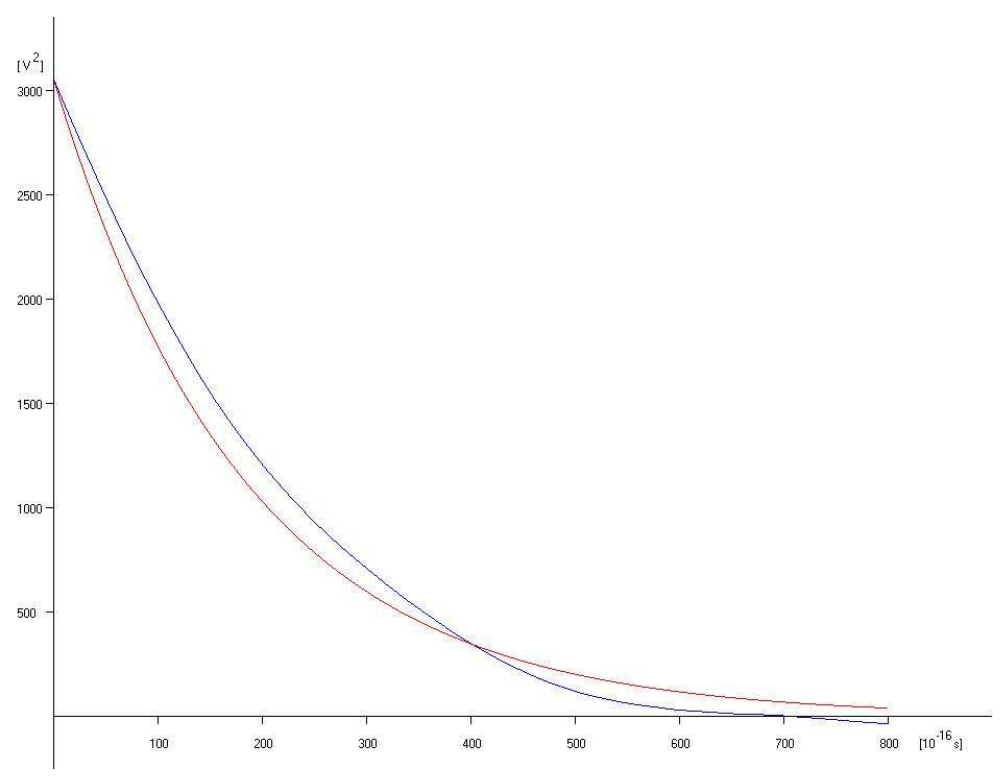

Figure 3: Parametric fit to the nonparametric estimate

interpreted as a typical frequency inherent in the signal; the obtained value is far beyond the capabilities of a contemporary oscilloscope, which makes the thermal voltage signal inaccessible for measuring devices. For a numerical illustration of $\widehat{c}$ we observe, however, that

$$
\widehat{c} \approx \frac{k_{B} \cdot T}{\hbar}=3.9276 \cdot 10^{13} \mathrm{~s}^{-1}
$$

holds where $\hbar$ denotes Planck constant.

Summarizing it can be stated that the simulated thermal voltage signal is statistically identified as a trajectory of a stationary Ornstein-Uhlenbeck process. Although we cannot claim the exactness of the modified Drude model for the description of a real nanoconductor, we believe that the latter would be a challenging object for the experimental study of thermal noise phenomena.

\section{Acknowledgments}

The authors would like to thank Georg Pflug from Vienna and Hajo Leschke from Erlangen for valuable comments on the first draft of the present contribution. 


\section{References}

[1] H. Bauer, Probability Theory, De Gruyter, Berlin, New York (1996).

[2] P. Billingsley, Probability and Measure, 3-rd Ed., Wiley, New York, Chichester (1995).

[3] E. Grycko, W. Kirsch, M. Könenberg, J. Li, T. Mühlenbruch, J. Rentmeister, Thermal noise in a modified Drude model, Int. J. Pure Appl. Math., 54, No. 4 (2009), 551-561.

[4] E. Grycko, W. Kirsch, T. Mühlenbruch, Amplification of thermal noise by an electrostatic field, Int. J. Pure Appl. Math., 60, No. 2 (2010), 187-192.

[5] E. Grycko, W. Kirsch, T. Mühlenbruch, Some quantum mechanical evidence for the amplification of thermal noise in an electrostatic field, Int. J. Pure Appl. Math., 69, No. 4 (2011), 437-507.

[6] P. Hall, P. Patil, Properties of nonparametric estimators of autocovariance for stationary random fields, Probab. Theory Relat. Fields, 99 (1994), 399424.

[7] J.D. Jackson, Classical Electrodynamics, 3-rd. Ed., John Wiley \& Sons, New York, Chichester, Weinheim (1999).

[8] E. Liebscher, Strong convergence of $\alpha$-mixing random variables with application to density estimation, Stoch. Proc. Appl., 65 (1996), 69-80.

[9] E. Liebscher, Asymptotic normality of nonparametric estimators under $\alpha$ mixing condition, Stat. Prob. Lett., 43 (1999), 243-250.

[10] E. Liebscher, Estimation of the density and the regression function under mixing conditions, Statistics \& Decisions, 19 (2001), 9-26.

[11] O. Moeschlin, E. Grycko, C. Pohl, F. Steinert, Experimental Stochastics, Springer-Verlag, Berlin-Heidelberg-New York (1998).

[12] O. Moeschlin, E. Grycko, Experimental Stochastics in Physics, SpringerVerlag, Berlin-Heidelberg-New York (2006).

[13] R. Müller, Rauschen, 2-nd Ed., Springer-Verlag, Berlin-Heidelberg-New York (1990).

[14] W. Nolting, Elektrodynamik, 6-th. Ed. Springer-Verlag, Berlin-HeidelbergNew York (2002). 
[15] M. Rosenblatt, Stationary Sequences and Random Fields, Birkhäuser, Boston-Basel-Stuttgart (1985).

[16] R.L. Wheeden, A. Zygmund, Measure and Integral, Marcel Dekker, New York, Basel (1977). 